\title{
GERENCIADOR DE REFERÊNCIAS MENDELEY: características e uso no contexto de um grupo de estudos de Pós- Graduação em Educação
}

\section{ISABEL MARIA SABINO DE FARIAS}

Pedagoga (UECE). Doutora em Educação Brasileira (UFC), com Estágio Pós-Doutoral pela Universidade de Brasília (UnB). Professora do Centro de Educação e do Programa de Pós-Graduação em Educação (PPGE) - Universidade Estadual do Ceará (UECE).

Líder do grupo de pesquisa Educação, Cultura Escolar e Sociedade (EDUCAS/CNPq). Coordenadora do Observatório Desenvolvimento Profissional Docente e Inovação Pedagógica.

ORCID: https://orcid.org/0000-0003-1799-0963.E-mail: isabel.sabino@uece.br

\section{RICARDO RODRIGUES DA SILVA}

Professor Assistente do Curso de Licenciatura em Ciências Biológicas - Faculdade de Educação, Ciências e Letras - FECLI/UECE, Iguatu/CE. Mestre em Ciências Veterinárias (UECE, 2004). Licenciado (2000) e Bacharel (2002) em Ciências Biológicas (UECE).

Professor da Rede Pública de Ensino do Estado do Ceará - Biologia e Química no Ensino Médio (2004-2008). Doutorando em Educação - Programa de Pós-Graduação em Educação da Universidade Estadual do Ceará - PPGE/UECE. Integrante do grupo de pesquisa Educação, Cultura Escolar e Sociedade - EDUCAS. ORCID: https://orcid.org/0000-0001-7199-2324.

E-mail: ricardo.rodrigues@uece.br

\section{SILVINA PIMENTEL SILVA}

Graduação em Educação da Universidade Estadual do Ceará - PPGE/UECE. Integrante do grupo de pesquisa Educação, Cultura Escolar e Sociedade - EDUCAS. ORCID: https://orcid.org/0000-0001-7199-2324. E-mail: ricardo.rodrigues@uece.br 


\section{GERENCIADOR DE REFERÊNCIAS MENDELEY: características e uso no contexto de um grupo de estudos de Pós-Graduação em Educação}

Este trabalho tem como objetivo discutir as principais características e funcionalidades do programa gerenciador de referências bibliográficas Mendeley, e apresentar um relato sobre uma ação formativa relacionada a esse software, realizada no contexto de um grupo de estudos de um Programa de Pós-graduação em Educação. Devido ao grande repertório de informações a que os pesquisadores educacionais têm acesso atualmente, pode ser benéfica a associação de dispositivos e recursos tecnológicos a ações investigativas. Nesse contexto, inserese o Mendeley, que é um gerenciador de referências que combina uma versão disponível para computadores, um aplicativo para dispositivos móveis e uma rede social on-line, apresentando diversas opções de leitura e organização de arquivos em PDF, além de anotações e destaques variados nos documentos e da busca de palavras e termos em todo o acervo de arquivos. A utilização do Mendeley demanda uma aprendizagem básica das suas principais funcionalidades. Nesse sentido, promoveu-se uma oficina sobre esse gerenciador de referências com integrantes de um grupo de estudos de Pós-Graduação em Educação. O primeiro momento da oficina consistiu em uma exploração teórica da temática; o segundo correspondeu à atividade prática em que os participantes fizeram uso do gerenciador Mendeley. Os participantes da oficina indicaram que o Mendeley lhes teria sido sugerido por colegas de grupo de estudos ou de pesquisas. Foram sinalizadas pelos participantes da oficina como características do Mendeley mais importantes: gratuidade, integração com programa editor de textos, armazenamento na nuvem e sincronização de dados de arquivos, integração com o sistema operacional do computador, criação de pastas para organização de referências e adição de citações diretamente nos textos. Quanto à organização/utilização de arquivos PDF, os principais aspectos do Mendeley assinalados pelos participantes foram: importação de documentos do computador e opções variadas para anotações, destaques e busca de PDFs no acervo. Verificamos que o Mendeley pode corresponder a um importante instrumento de apoio ao desenvolvimento de atividades acadêmicas de pós-graduandos. Comprovamos que os pós-graduandos devem ser encorajados a usar softwares como o Mendeley enquanto escrevem seus trabalhos acadêmicos e artigos científicos, pois o hábito de uso desses programas não apenas tornará a vida dos pesquisadores mais fácil, mas também auxiliará a superar tensões que se apresentam quando se faz necessário revisar e editar os textos produzidos.

Palavras chave: Ferramenta digital. Gerenciamento de referências. Mendeley. Pós-graduandos.

\section{MENDELEY REFERENCE MANAGER: characteristics and use in the context of a study group of the Postgraduate in Education}

This article aims to discuss the main characteristics and functionalities of the Mendeley reference management program and to present an account of a formative action related to this software, carried out in the context of a study group of the Post- Graduation in Education Program. Due to the large repertoire of information that educational researchers currently have access to, it may be beneficial to associate devices and technological resources with investigative actions. In this context, Mendeley, a reference manager that combines a version available for computers, a mobile app and an online social network, has several options for reading and organizing PDF files, as well as annotations and varied highlights in the documents and the search of words and terms throughout the archives collection. The use of Mendeley requires a basic learning of its main functionalities. In this sense, a workshop on this manager of references was promoted with members of a study group of the Post-Graduation in Education. The first moment of the workshop consisted in a theoretical exploration of the theme; the second corresponded to the practical activity in which participants made use of the Mendeley manager. The workshop participants indicated that Mendeley would have been suggested to them by study group or research group

P/U $a i j S$ Salvador, v. 4, n.2, p. 65-79, mai./ago. 2019 
colleagues. The workshop participants also flagged as the most important features of Mendeley: gratuity, text editor integration, cloud storage and file data synchronization, integration with the computer operating system, creation of folders for organization of references and addition of citations directly in the texts. Regarding the organization / use of PDF files, the main aspects of Mendeley pointed out by the participants were: import of computer documents and varied options for annotations, highlights and search of PDFs in the collection. It was verified that Mendeley can be an important tool to support the development of academic activities of postgraduation students. It was proved that the post-graduate students should be encouraged to use softwares as Mendeley while writing their academic papers and scientific articles, because the habit to use those programs will not only make the life of the researchers easier, but also help to overcome tensions that arise when it is necessary to review and edit the produced texts.

Keywords: Digital tool. Reference management. Mendeley. Postgraduate students.

\section{GERENCIADOR DE REFERENCIAS MENDELEY: características y uso en el contexto de un grupo de estudios de Postgrado en Educación}

Este trabajo tiene como objetivo discutir las principales características y funcionalidades del programa gestor de referencias bibliográficas Mendeley y presentar un relato sobre una acción formativa relacionada a ese software, realizada en el contexto de un grupo de estudios de un Programa de Postgrado en Educación. Debido al gran repertorio de informaciones a que los investigadores educacionales tienen acceso actualmente, puede ser beneficioso la asociación de dispositivos y recursos tecnológicos a acciones investigativas. En este contexto, se inserta Mendeley, que es un gestor de referencias que combina una versión disponible para ordenadores, una aplicación para dispositivos móviles y una red social on-line, presentando diversas opciones de lectura y organización de archivos en PDF, además de notas y destaques variados en los documentos y de la búsqueda de palabras y términos en todo el acervo de archivos. La utilización de Mendeley demanda un aprendizaje básico de sus principales funcionalidades. En ese sentido, se promovió un taller sobre ese gestor de referencias con integrantes de un grupo de estudios de Postgrado en Educación. El primer momento del taller consistió en una exploración teórica de la temática; el segundo correspondió a la actividad práctica en que los participantes hicieron uso del gestor Mendeley. Los participantes del taller indicaron que Mendeley les habría sido sugerido por colegas de grupo de estudios o de investigaciones. Se han señalado por los participantes del taller como características de Mendeley más importantes: gratuidad, integración con programa editor de textos, almacenamiento en nube y sincronización de datos de archivos, integración con el sistema operativo del ordenador, creación de carpetas para organización de referencias y adición de carpetas citas directamente en los textos. En cuanto a la organización / utilización de archivos PDF, los principales aspectos de Mendeley señalados por los participantes fueron: importación de documentos del ordenador y opciones variadas para anotaciones, destaques y búsqueda de PDF en el acervo. Verificamos que Mendeley puede corresponder a un importante instrumento de apoyo al desarrollo de actividades académicas de posgraduados. Hemos comprobado que los posgraduados deben ser alentados a usar software como Mendeley mientras escriben sus trabajos académicos y artículos científicos, pues el hábito de uso de esos programas no sólo hará la vida de los investigadores más fácil, pero también ayudará a superar las tensiones que se presentan cuando se hace necesario revisar y editar los textos producidos.

Palabras clave: Herramienta digital. Gestión de referencias. Mendeley. Pos graduandos. 


\section{GERENCIADOR DE REFERÊNCIAS MENDELEY: características e uso no contexto de um grupo de estudos de Pós- Graduação em Educação}

\section{Introdução}

A realização de uma pesquisa consistente no campo educacional tem em sua base uma ampla e sofisticada revisão de literatura. Para tanto, os estudiosos da educação devem possuir um variado repertório de habilidades e conhecimentos que lhes permita analisar e sistematizar as informações provenientes das produções científicas inerentes às suas pesquisas.

Considerando o grande repertório de informações a que os pesquisadores educacionais têm acesso atualmente, é primordial que sejam estabelecidos procedimentos metodológicos e técnicos para otimizar o trabalho com esse material. As inovações tecnológicas inserem-se nesse panorama, de modo que podem contribuir para a elaboração de uma revisão bibliográfica mais ampla e, ao mesmo tempo, mais consistente. Isso se deve às ferramentas de busca e de organização de achados cada vez mais eficientes e ao acesso aos documentos em formatos digitais como artigos, trabalhos publicados em eventos, livros, reportagens etc. (RIEDNER; PISCHETOLA, 2016).

Em vista das pesquisas delineadas nos Programas de Pós-graduação em Educação, tais competências têm sua relevância destacada, tendo em vista a complexidade das temáticas investigadas e a grande quantidade de informações sobre as quais os pesquisadores dedicam esforço e tempo para organização, leitura e análise. Com base nesse pressuposto, este escrito objetiva discutir as principais características e funcionalidades do programa gerenciador de referências bibliográficas Mendeley, análise entrecruzada com relatos de participantes de uma ação formativa sobre esse software, realizada no contexto de um grupo de estudos de um Programa de Pós-Graduação em Educação.

\section{Recursos tecnológicos e ações investigativas na pesquisa educacional}

As profundas transformações comunicacionais verificadas na sociedade contemporânea são consequências de avanços sem precedentes nos campos relacionados às tecnologias. Tais modificações afetam a vida das pessoas de variadas maneiras - no contexto social, político, econômico, cultural etc. 
A modalidade atual de acesso interativo às informações é, muitas vezes, mediada e ampliada pela utilização de recursos e dispositivos tecnológicos com as mais diversificadas funções e finalidades. De acordo com Lévy (2004), estamos presenciando a reorganização de várias relações na sociedade contemporânea, o que tem impacto sobre procedimentos investigativos acadêmicos:

Novas maneiras de pensar e de conviver estão sendo elaboradas no mundo dastelecomunicações e da informática. As relações entre os homens, o trabalho e a própria inteligência dependem, na verdade, da metamorfose incessante de dispositivos informacionais de todos os tipos. Escrita, leitura, visão, audição, criação, aprendizagem são capturados por uma informática cada vez mais avançada. Não se pode mais conceber a pesquisa científica sem uma aparelhagem complexa que redistribui as antigas divisões entre experiência e teoria. Emerge, neste final do século XX, um conhecimento por simulação que os epistemologistas ainda não inventariaram (LÉVY, 2004, p. 7).

Cada vez mais, a tecnologia adquire um papel de destaque no meio científico, mas sempre estando sujeita às pressões do curso histórico. A evolução tecnológica, ao mesmo tempo em que influencia e absorve desdobramentos das modificações inter-relacionais, simboliza o progresso humano situado em meio à sociedade informacional (CLAUDINO, 2013).

As novidades trazidas pelas tecnologias da informação e comunicação (TICs) são bemvindas na esfera universitária, onde suas contribuições podem se dar de várias formas. No campo educacional, estratégias acadêmicas promissoras podem emergir a partir da aproximação com as ferramentas tecnológicas.

Obviamente, esse movimento de mudança para integração de inovações tecnológicas no contexto educacional não pode ocorrer de modo desconectado da realidade social. Conforme relatam Gatti e Barretto (2009), inovação de práticas não possuem uma fórmula pronta para aplicação; ao contrário, apresentam dependência de particularidades institucionais, pedagógicas, sociais e culturais do campo específico no qual ela se enquadra, aspecto também reforçado por Farias (2006).

Deve-se salientar, ainda, que a integração dessas novas práticas mediadas por processos tecnológicos pode acontecer de modo bastante desigual, quando considerados ambientes sociais distintos. Enquanto ela se dá de modo rápido na comunicação midiática - onde os agentes envolvidos promovem sua incorporação e utilização em uma lógica de mercado, tende a ser mais lenta nos processos educacionais - cujas particularidades organizacionais impõem dificuldades a mudanças institucionais e pedagógicas acarretadas pela integração de novos dispositivos 
tecnológicos. Outro obstáculo que também precisa ser superado diz respeito à desigualdade no acesso e na apropriação das tecnologias digitais conforme as classes sociais e as regiões do planeta (BÉVORT; BELLONI, 2009).

A despeito dessas e de outras eventuais situações que precisam ser contornadas no que se refere à incorporação de práticas intermediadas por processos tecnológicos no campo educacional, é inegável o leque de possibilidades que se abre quando essa aproximação realmente acontece, como mencionado por Nunes (2010):

O campo da pesquisa em Ciências Sociais e Humanas e, particularmente, em Educação, beneficia-se de forma incisiva dos avanços tecnológicos. A convergência digital tem proporcionado mudanças na forma de fazer ciência, com repercussões no crescimento quantitativo e qualitativo da produção e acesso ao saber (NUNES, 2010, p. 21).

De fato, o aumento da disponibilização de dados disponíveis digitalmente verificado nos últimos anos sinaliza a necessidade de que os pesquisadores obtenham alternativas ágeis e apropriadas para gerenciar essas informações. Uma possibilidade para aprimorar esse processo é a utilização de ferramentas computacionais específicas, quetêm se tornado elementos relevantespara colaborar com as atividades de usuários que possuem essas demandas que, com frequência, examinam criteriosamente a produção científica correlacionada (YAMAKAWA et al., 2014). Nesse cenário, verifica-se que podem emergir importantes benefícios da associação entre recursos tecnológicos e ações investigativas no campo da pesquisa educacional, como o levantamento e organização de informações acerca das produções acadêmicas.

A análise da literatura produzida na área da temática investigada auxilia o pesquisador na definição do amplo contexto e na demarcação clara dos limites conceituais da pesquisa, além de colaborar com a sua tomada de decisões para desenvolvimento da investigação. Uma boa revisão da literatura constitui-se em base de sofisticação teórica e metodológica, aprimorando a qualidade e a utilidade da pesquisa subsequente (BOOTE; BEILE, 2016).

A crescente disponibilização de informações nos ambientes digitais representa o surgimento de novas fontes de dados para pesquisadores e, ao mesmo tempo, um desafio para organização desse material, conforme relatam Yamakawa et al. (2014):

[...] uma maior facilidade de acesso àsdiversas fontes de conhecimento vem se consolidando, especialmente por meio da Web, dos portais e das bases de 
periódicos. [...] Somado a isso, com o passar do tempo, a produção contínua e constante do conhecimento acaba por dificultar a tarefa do pesquisador, uma vez que o volume de publicações e conteúdos se amplia consideravelmente (YAMAKAWA et al., 2014, p. 168).

Diversos cientistas já fazem uso de ferramentas computacionais para otimizar a busca e a organização do seu repertório de informações. Esses programas computacionais que possibilitam o gerenciamento de referências bibliográficas, dentre outras funções, possibilitam o arquivamento e a organização de trabalhos, bem comofacilitam a sua citação pelos pesquisadores. A obtenção de informações sobre essas produções científicasem bases de dadosonline somadas àquelas que podem ser incorporadas diretamente pelo pesquisador nesses recursos digitais tornam possível a geração automática da referência bibliográfica integral no final do documento de texto (MULDROW; YODER, 2009).

É preciso ressaltar que é atribuição do pesquisador realizar a validação do atendimento aos critérios objetivos e subjetivos de cientificidade e confiabilidade do acervo a ser gerenciado pela ferramenta digital. Em outras palavras, não se pode esperar que o uso de recursos computacionais represente por si mesmo a garantia de qualidade científica das publicações que são gerenciadas.

Deve-se destacar, ainda, que o gerenciamento de citações é apenas um aspecto do processo de pesquisa mais amplo. Esse processo tem sido estudado extensivamente no tocante a estudantes de graduação, mas não tanto para estudantes de pós-graduação e professores (EMANUEL, 2013).

\section{Características e funções do software de gerenciamento de referências Mendeley}

Atualmente, existem diversas ferramentas digitais que podem contribuir com o trabalho do pesquisador no que se refere à catalogação e organização de arquivos eletrônicos de referências bibliográficas. Neste trabalho, centralizamos nossa atenção sobre aspectos do Mendeley (Figura 1), um dos gerenciadores de referências mais populares entre pesquisadores (CARREÑO, 2014).

O Mendeley é um software gerenciador de referências, produzido pela Elsevier, para gerenciar e compartilhar documentos de pesquisa. Ele combina o Mendeley Desktop - disponível para computadores Windows, macOS e Linux -, o aplicativo Mendeley para aparelhos móveis disponível para Android e iOS - e a Mendeley Web, uma rede social on-line para pesquisadores (http://www.mendeley.com). O software está disponível para download, ou para acesso pelo site do programa, apenas em língua inglesa. O Mendeley apresenta diversas opções de leitura 
e organização de arquivos em PDF. Também possibilita a realização de anotações e destaques variados nos documentos, além da busca de palavras e termos em todo o acervo de arquivos (Figura 1).

Figura 1. Visualização do Mendeley Desktop.

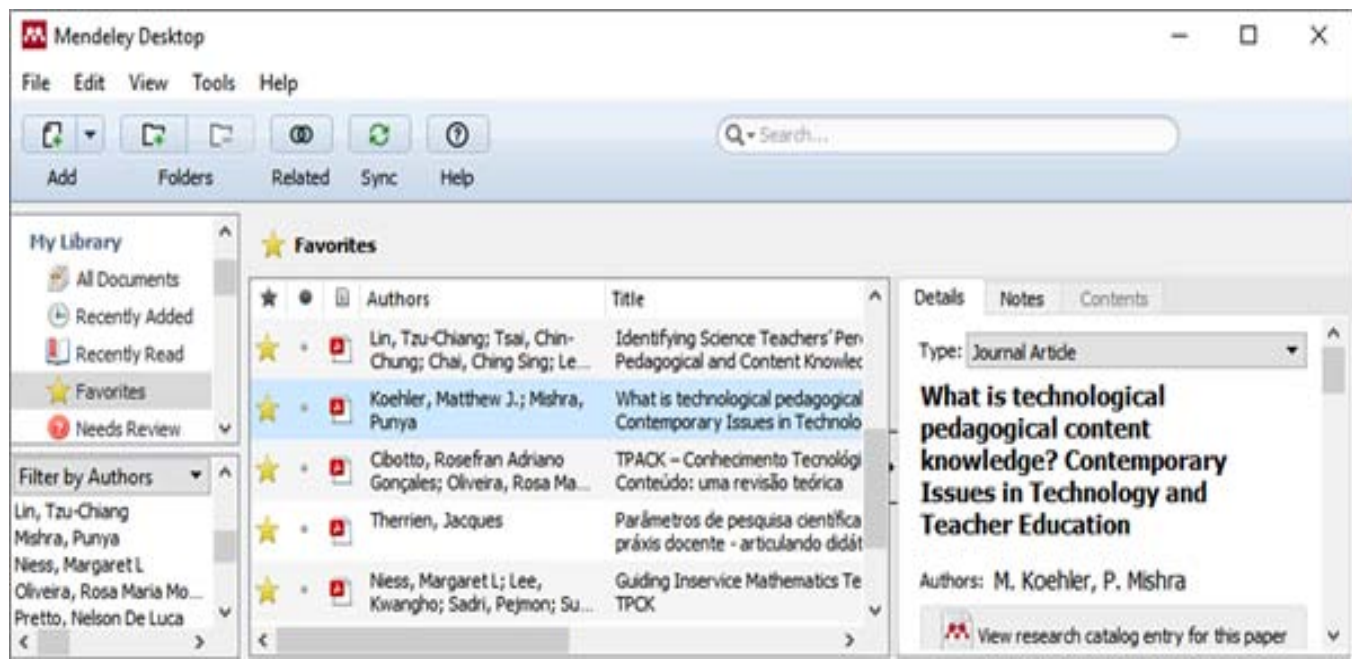

Fonte: Elaborado pelos autores, 2019.

O programa foi denominado de Mendeley em homenagem a dois cientistas: Dmitri Ivanovich Mendeleyev (ou Mendeleev) - que desenvolveu a tabela periódica de elementos químicos - e Gregor Mendel - considerado o "pai da genética moderna". Assim como Gregor Mendel estudou a herança de traços nas plantas, o Mendeley permite que o pesquisador rastreie como ideias e teorias acadêmicas evoluem e se polinizam entre si. Dmitri Mendeleyev formou a tabela periódica com base nas propriedades dos elementos conhecidos, depois usou esses dados para prever as propriedades dos elementos a serem descobertos; de modo análogo, o Mendeley auxilia o pesquisador a descobrir novas literaturas baseadas nos elementos conhecidos de sua biblioteca (HENNING, 2008).

O Mendeley é disponibilizado em uma versão premium paga e em uma versão básica que é gratuita, mas requer registro. Além disso, o Mendeley exige que o usuário armazene todos os dados básicos de citação em seus servidores, ficando o armazenamento de cópias de 
documentos a critério do usuário. Após o registro, o Mendeley fornece ao usuário 2 GB de espaço de armazenamento gratuito na web - a versão paga do programa amplia essa capacidade de armazenamento.

Existem recursos adicionais do Mendeley que proporcionam a sua integração com outros recursos digitais. A extensão Web Importer pode ser integrada ao navegador de internet (Chrome, Firefox, Safari e Internet Explorer). Ela é integrada ao navegador na parte superior direita em forma do logotipo do Mendeley; ao se clicar nele, a página ou documento em questão é encaminhado para a sua biblioteca do Mendeley. O Citation Plugin pode ser instalado no computador para que, ao realizar seus trabalhos utilizando o programa editor de textos (Microsoft Worde LibreOffice), o pesquisador consiga exportar citações e referências dos arquivos da sua biblioteca do Mendeley.

Uma das características muito úteis do Mendeley, considerando o cenário tecnológico cada vez mais interconectado da atualidade, é a possibilidade de sincronização dos documentos eletrônicos arquivados no programa com o sistema de armazenamento on line. Outro aspecto distintivo do Mendeley é sua funcionalidade de recuperar metadados dos arquivos PDFs das referências usadas para a constituição do material (MARCHIORI et al., 2010).

O uso do Mendeley permite ao pesquisador manter o acervo dos documentos do seu computador em sincronia com o acervo arquivado nos servidores da internet. Isso garante ao pesquisador a possibilidade de acesso, por meio da internet, aos dados que tenham sido por ele selecionados - o que representa um componente extra de segurança para seu acervo eletrônico.

O uso do Mendeley possibilita o acesso ao acervo de informações pela internet, tendo ou não Mendeley Desktop instalado. Vale ressaltar que o Mendeley Desktop pode ser instalado em computadores distintos, mediante acesso àinternet para sincronização com a conta web. A biblioteca pessoal também pode ser acessada pela conta individual na página eletrônica do software, caso a versão Desktop não esteja instalada no computador em uso. Outra possibilidade para alcance do conjunto de arquivos pessoais é através do uso do aplicativo móvel (YAMAKAWA et al., 2014).

Santos, Santos e Lima (2018) ressaltam que os três principais gerenciadores de referências disponíveis atualmente no mercado são EndNote, Zotero e Mendeley, sendo este último o de criação mais recente. Os autores afirmam que, dentre esses gerenciadores de referências, o Mendeley se destaca pela quantidade e qualidade de recursos que podem ser utilizados no gerenciamento e compartilhamento de referências bibliográficas. Por essas razões, o Mendeley tem despertado cada vez mais a atenção de pesquisadores brasileiros. 
A despeito do Mendeley possibilitar ao pesquisador a otimização dos processos de organização e acesso aos arquivos de referências, a sua utilização demanda uma aprendizagem básica das principais funcionalidades.

Tendo em mente que muitos pós-graduandos desconhecem a existência de ferramentas digitais como o Mendeley, promoveu-se a realização da oficina Mendeley: gerenciador de referências e rede social acadêmica no âmbito do Grupo de Pesquisa Educação, Cultura Escolar e Sociedade (EDUCAS), equipe cadastrada no Diretório de Grupos de Pesquisa do CNPq e vinculada Programa de Pós-Graduação em Educação (PPGE) da Universidade Estadual do Ceará (UECE). A seguir, apresentamos um relato a respeito da ação formativa desenvolvida.

\section{Percurso metodológico da ação formativa}

A ação formativa sobre o Mendeley foi realizada no dia 06 de junho de 2019, no Laboratório de Informática do Centro de Educação (CED) da Universidade Estadual do Ceará (UECE), contando com a participação de 7 integrantes do EDUCAS/UECE/PPGE. Este momento formativo teve como propósito promover aprendizagem básica das principais funcionalidades do Mendeley entre pós-graduandos ligados ao Núcleo "Desenvolvimento Docente, Currículo e Inovação" da linha de pesquisa "Formação e Desenvolvimento Profissional em Educação" do PPGE/UECE, de modo que pudessem considerá-lo como um instrumento para auxílio em seus trabalhos acadêmicos. Os responsáveis pelo processo de formação foram dois doutorandos desse programa, ambos ligados a linha de pesquisa supra citada.

A ação formativa foi estruturada contemplando três momentos distintos. O primeiro consistiu em uma exploração teórica da temática; o segundo correspondeu à atividade prática em que os participantes fizeram uso do gerenciador de referências Mendeley; o terceiro versou sobre a explicitação das contribuições da formação para os participantes.

O primeiro momento foi marcado pela discussão a respeito dos tópicos: apresentação do Mendeley e de suas principais funcionalidades; procedimentos para instalação do Mendeley; criação de biblioteca pessoal; organização de pastas e arquivos das referências; procedimentos para realizar citação de referências no editor de textos Word.

O segundo momento assumiu uma feição de oficina, ocasião em que os participantes tiveram a oportunidade de realizar algumas ações práticas com o Mendeley, mediante uso de seus computadores pessoais, dentre as quais destacamos: criação de conta online; instalação do Mendeley Desktop; importação de referências bibliográficas da internet; importação de referências 
bibliográficas a partir de arquivos PDF; realização de anotações e destaques em documentos; instalação do plugin Web Importer; instalação do Citation Plugin; edição de metadados de arquivos PDF; inserção de citações em um documento do Word; inserção de bibliografia em um documento do Word; criação de grupos de trabalho.

Importa registrar que a ação formativa aqui descrita, delineada e desenvolvida com base na demanda dos participantes do grupo de pesquisa EDUCAS, inspirou-se nos pressupostos que modelam uma investigação-ação. Essa modalidade de pesquisa é identificada como um tipo de investigação no qual o investigador se envolve ativamente na causa da investigação. Tanto procedimentos qualitativos como quantitativos podem ser utilizados na investigaçãoação (BOGDAN; BIKLEN, 1994). Assim, além do interesse e necessidade de aprender a usar o Mendeley evidenciadas pelos participantes do EDUCAS, fundamental no desenho da ação formativa, o terceiro momentoconsubstanciou-se na realização da observação durante a oficina e no emprego do questionário como instrumento de produção de dados após a realização da atividade formativa. A observação realizada auxiliou no delineamento do teor do questionário.

Foi utilizada a ferramenta Formulários Google para elaboração do questionário (com perguntas abertas e fechadas), que foi enviado eletronicamente para os participantes da oficina, após a sua realização. $O$ intuito do uso do questionário foi identificar as percepções dos participantes quanto à vivência da atividade formativa e quanto às eventuais contribuições do Mendeley para seus trabalhos acadêmicos.

\section{Perspectivas dos partcipantes sobre o Mendeley}

Participaram da ação formativa 7 pós-graduandos integrantes do EDUCAS, sendo 5 mestrandos e 2 doutorandos. Os participantes indicaram estarem muito satisfeitos quanto às atividades realizadas nessa atividade, à atuação dos ministrantes e ao atendimento das suas expectativas. Também assinalaram elevada satisfação quanto às contribuições da ação formativa para o desenvolvimento de suas atividades acadêmicas.

Com relação à metodologia utilizada, os participantes mostraram-se agradados, conforme pode ser verificado nas falas:

Os mediadores da oficina mostraram-se muito disponíveis para tirar as dúvidas e apresentar os benefícios do uso do Mendeley. Foi excelente. (PARTICIPANTE A). 
A metodologia utilizada promoveu uma ampla participação e envolvimento dos participantes através de atividades práticas e bem orientadas (PARTICIPANTE C).

Os participantes destacaram a importância da colaboração dos pares na pesquisa acadêmica, uma vez que indicaram que o Mendeley lhes teria sido sugerido por colegas de grupo de estudos ou de pesquisas.

Com relação às características gerais do Mendeley, foram indicadas como as mais importantes pelos participantes da ação formativa: gratuidade, integração com o editor de textos Word, armazenamento na nuvem e sincronização de dados de arquivos, integração com o sistema operacional do computador, criação de pastas para organização de referências e adição de citações diretamente nos textos.

No que diz respeito à organização de arquivos PDF, as principais características do Mendeley assinaladas pelos participantes foram: importação de documentos do seu computador, realização de anotações ou de destaques em PDFs e pesquisa de texto no acervo completo da biblioteca pessoal.

Os aspectos observados nesse estudo estão em consonância com a percepção de Emanuel (2013) de que os usuários preferem ferramentas de gerenciamento de citações e referências que possam importar, organizar e citar itens da maneira mais eficiente possível, dando menor ênfase em outros recursos, muitas vezes relacionados a aspectos sociais.

A impressão geral dos participantes da ação formativa sobre o Mendeley foram positivas: "Um ótimo auxílio acadêmico" (PARTICIPANTE A), "É uma ferramenta que facilita bastante uma das atividades do pesquisador" (PARTICIPANTE C). Alguns participantes, contudo, destacaram como aspectos negativos do gerenciador: "A relativa restrição de documentos apenas em PDF" (PARTICIPANTE B), "O fato de ser no idioma inglês" (PARTICIPANTE D).

Os participantes relataram que, após a realização da ação formativa, passaram a manifestar o interesse em utilizar o Mendeley em suas atividades acadêmicas, como ilustrado pelo participante E:

Gostei muito da metodologia adotada pois os palestrantes conseguiram atender todos os alunos com diferentes dúvidas e habilidades no uso da tecnologia. [...] Achei muito bom. Estou ainda me apropriando de suas funções, mas já uso 
em atividades básicas para organizar referências, leituras com marcações e citações (PARTICIPANTE E).

Com relação às características gerais do Mendeley, foram indicadas como as mais importantes pelos participantes da ação formativa: gratuidade, integração com o editor de textos Word, armazenamento na nuvem e sincronização de dados de arquivos, integração com o sistema operacional do computador, criação de pastas para organização de referências e adição de citações diretamente nos textos.

\section{Considerações finais}

A análise buscou evidenciar que ações formativas centradas na promoção de aprendizagens básicas em relação a ferramentas digitais podem contribuir para o pós-graduando em seu percurso de formação como pesquisador, sobretudo na área educacional. Iniciativas nessa direção tornamse mais relevante se considerarmos que existe desconhecimento por parte desses pesquisadores da existência desses recursos eletrônicos e de suas funcionalidades.

A oportunidade de aprendizagem vivenciadas por mestrandos e doutorandos revelou-se, ainda, potente para estimular o interesse em incluir o Mendeley como um instrumento para auxiliar as suas práticas investigativas.

Os elementos evidenciados permitem firmar a proposta da ação formativa como bem sucedida, oferecendo aos pós-graduandos um instrumento de apoio ao desenvolvimento de suas atividades acadêmicas.

Acreditamos que os pós-graduandos devem ser encorajados a usar softwares como o Mendeley enquanto escrevem seus trabalhos acadêmicos e artigos científicos. O desenvolvimento do hábito de uso desses programas não apenas tornará a vida dos pesquisadores mais fácil, mas também auxiliará a superar tensões que se apresentam quando se faz necessário revisar e editar os textos produzidos.

\section{REFERÊNCIAS}

BÉVORT, E.; BELLONI, M. L. Mídia-educação: conceitos, história e perspectivas. Educação e Sociedade, 30 (109), 1081-1102. Disponível em: <http://www.scielo.br/pdf/es/v30n109/ v30n109a08.pdf>. Acesso em: 18 set. 2017. 
BOGDAN, Roberto C.; BIKLEN, Sari Knopp. Investigação qualitativa em educação: uma introdução à teoria e aos métodos. Porto: Porto Editora, 1994.

BOOTE, David N.; BEILE, Penny. ResearchPreparationFeaturesi Scholars Before Researchers: On the Centrality of the Dissertation Literature Review in Research Preparation. EducationalResearcher, v. 34, n. 6, p. 3-15, 2016.

CARREÑO, Montserrat López. Análisis comparativo de los gestores bibliográficos sociales Zotero, Docear y Mendeley: características y prestaciones. Cuadernos de Gestión de Información, v. 4, p. 51-66, 2014.

CLAUDINO, Osmundo Rocha. Educar na sociedade da informação. Reflexão e Ação, v. 21, n. 2, p. 49-72, 2013. Disponível em: <https://online.unisc.br/seer/index.php/reflex/article/ view/3437>. Acesso em: 3 ago 2018.

EMANUEL, Jenny. Usersandcitation management tools: use andsupport. Reference Services Review, v. 41, n. 4, p. 639-659, 2013.

FARIAS, Isabel Maria Sabino de. Inovação, Mudança e Cultura Docente. Brasília: Liber Livro, 2006.

GATTI, Bernardete Angelina; BARRETTO, Elba Siqueira de Sá (Coords.). Professores do Brasil: impasses e desafios. Brasília: UNESCO. 2009.

HENNING, Victor. Howourname evolved from B-moviemonsterto Mendeley, 2008. Disponível em:, <https://blog.mendeley.com/2008/04/23/how-our-name-evolved-from-b-movie-monster-to-mendeley/>. Acesso em: 12 jun. 2019.

LÉVY, Pierre. As tecnologias da inteligência - o futuro do pensamento na era da informática. Tradução Carlos Irineu da Costa: Editora 34, 2004.

MARCHIORI, Patrícia Zeni et al. Aspectos estruturais e motivacionais e possíveis zoneamentos discursivos em software social acadêmico. Liinc em Revista, v. 6, n. 2, p. 355-369, 2010. Disponível em: $<$ http://revista.ibict.br/liinc/article/view/3245/2879>.

MULDROW, Jason; YODER, Stephen. Out of cite! Howreference managers are takingresearchtothenextlevel. Political Science and Politics, v. 42, n. 1, p. 167-172, 2009.

NUNES, João Batista Carvalho. Busca científica na pesquisa em Educação: tendências atuais. In: FARIAS, I. M. S. de; NUNES, J. B. C.; NÓBREGA-THERRIEN, S. M. Pesquisa científica 
para iniciantes: caminhando no labirinto. Fortaleza: EdUECE, 2010 (vol. 1).

RIEDNER, Daiani Damm Tonetto; PISCHETOLA, Magda. Tecnologias Digitais no Ensino Superior: uma possibilidade de inovação das práticas? Educação, Formação \& Tecnologias, v. 9, n. 2, p. 37-55, 2016. Disponível em: <http://eft.educom.pt>.

SANTOS, Francisco Edvander Pires; SANTOS, Izabel Lima dos; LIMA, Juliana Soares. Treinamento a distância sobre o gerenciador de referências Mendeley. 2018, Salvador: [s.n.], 2018. p. 917-932.

YAMAKAWA, Eduardo Kazumi et al. Comparativo dos softwares de gerenciamento de referências bibliográficas: Mendeley, End Note e Zotero. TransInformação, v. 26, n. 2, p. 167-176, 2014.

Enviado em: 10 de julho de 2019

Apreciado em: 30 de julho de 2019

Inserido em: 01 de agosto de 2019 